Ind alsı keine klare Ansicht über ihr Wesen gewinnen konnte. Und doch liegt es so nahe, einen terrestrischen Lichtstrahl Is das Bruchstïck cines cülestischen anzusehen, und die terestrische Refraktion als ein Bruchstück der astronomischen a betrachten. Ich werde mit ein par Worten den Werth :iner solchen Auffassung der terrestrischen Refralition nachaweisen versuchen. Sei $A B S$ die Kurve eines Lichtstrabls, ler von einem Gestime $S$ herkommend in die Atmosphïre ritt, um iiber einen irdischen Punkt $B$ nach dem Ende $A$ les Strahls zu gelangen, so ist, wenn $R_{1}$ und $R_{2}$ die astroromische Strahlenlrechung des $S$ in $A$ und $B$ bezeichnen, die rdische Sirahienbrechung des Punktes $B$ in $A$, oder $A$ in $B$ : $=\frac{K_{1}-K_{22}}{2}$, wenn in $A$ drr Punkt $B$ einen Höhenwinkel ildet. Für den Fall aber, wo $B$ aus $A$ und $A$ aus $B$ unter inem Tiefenwinkel erscheint, ist $r=R_{3}-\frac{R_{1}+R_{2}}{2}$, wenn
$R_{3}$ den Werth der astronomischen Strahlenbrechung (in der Horizontale) für denjenigen Punkt zwischen $A$ und $B$ bezeichnet, wo der Strahl der Erdoberfläche parallel geht. Eive einfache Betrachtung der bildlichen Darstellung dessen, was hier in wenigen Buchstaben und Worten niedergelegt ist, wird die mathematische Schärfe des Resultats darthun. Der IÖ̈henwinkel in $A=a$ gesetzt, giebt ihn für $B=a+0,91 . c$ mit hinreichenter Genauigkeit für irdische Distanzen; $c$ aber ist der Centriwinkel im Mittelpunkte der Erdc für den Bogen $A B$. Mit Ilülfe astronnmischer Strahlenbrechung, begleitet von den beobachteten oder berechneten meteorologischen Daten ziveier terrestrischer Punkte $A$ und $B$, kann man folglich mit Leichtigkeit die terrestrische Refraktion des $A$ in $B$ und des $B$ in $A$ sehr angenähert finden.

Eglisau, im Kanton Zürich, den $30^{\text {sten Mai } 1842 .}$

Hs. Heinrich Denzler.

Schreiben des Herrn Hofraths Mädler, Directors der Dorpater Sternwarte, an den IIerausgeber. Dorpat 1842. Ipril 10.

[ch habe „die Bahnbewegungen der Doppelsterne“‘ zum Thema iner Abhandlung gewählt, die mit dem unter der Presse beindlichen $9^{\text {ten }}$ Bande der Dorpater Beobachtungen erscheinen vird. Die Zahl derer, bei dewen eine Bewegung dieser Art nit Sicherheit zu erkennen ist, steigt gegenwärtig schon auf

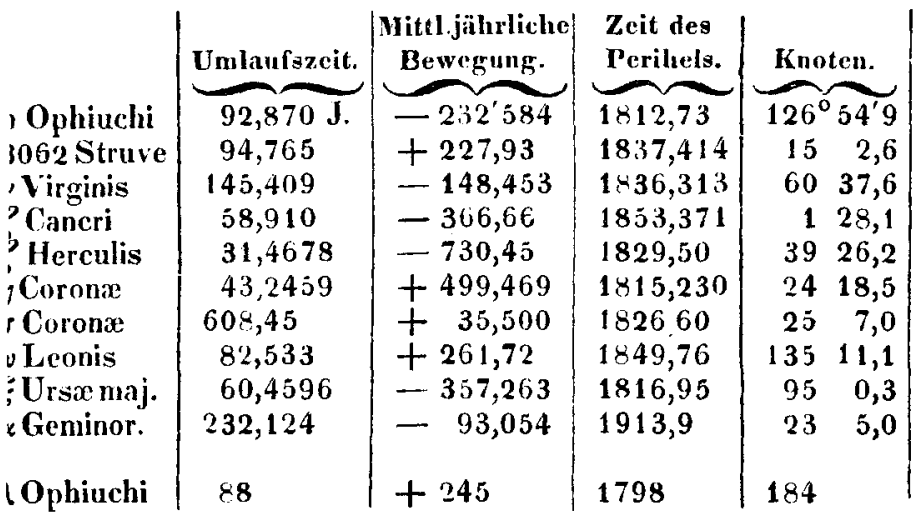

Yür die meisten dieser Bahnen lagen bereits frübere Versuche tor; aber alle sind aufs noue untersucht, nur $\xi$ Ursa majoris, sei welchem auch die neuesten Beobachtungen noch sehr gut ibereinstimmen, ist unverändert so angenommen, wie ich die Bahn 1836 gegeben habe.

Bereits dreimal habe ich in gegenwärtigem Frühjahr teleskopische Steruschnuppen durch das Feld des Refraktors zichen sehen. So wenig nun silche isolirte Wahrnehmungen für specielle Berechnungen dienen können, so
110 , und für eimen kleinen Theil derselben konnte der Versuch gemacht werden, die Elemente ciner Kepicrsclien Ellipse abzuleiten. Ich gebe hier vorläufig dirse Elemente selbst und verweise wegen des näheren Đetails auf die erwähnte $\mathrm{Ab}$ Laudlung.

\begin{tabular}{|c|c|c|c|c|c|}
\hline & Perihel vom & Excentri- & Halbe grofse & & \\
\hline Neigung. & Knoten. & cinât. & Axe. & $\boldsymbol{M}^{\frac{1}{3}} \pi$ & \\
\hline$t^{\prime} 4$ & $142^{\circ} 528$ & 0,44380 & 4192 & $020+41$ & \\
\hline $35 \quad 31,3$ & 13527,4 & 0,44958 & 1,255 & 0,06038 & \\
\hline $2+39,2$ & $78 \quad 22,1$ & 0,86815 & 3,492 & 0,12300 & \\
\hline 6316,8 & 266 & 0,23486 & 1,2922 & 0,08515 & \\
\hline $50 \quad 32,7$ & 262 & 0,45454 & 1,189 & 0,1193 & \\
\hline 7,7 & 26120,6 & 0,33760 & 1,0879 & 0,08931 & \\
\hline 2929,1 & 6428,3 & 0,69978 & 3,918 & 0,05456 & sehr unsicher. \\
\hline $46 \quad 33,5$ & $185 \quad 27,2$ & 0,64338 & 0,857 & 0,04520 & \\
\hline $52 \quad 15,5$ & 12940,5 & 0,40368 & 2,290 & 0,1487 & \\
\hline 7058,3 & $87 \quad 36,8$ & 0,79725 & 7,008 & 0,1860 & \\
\hline $45^{\circ}$ & 0 & 0,37 & 1,1 & 0,05 & läufig. \\
\hline
\end{tabular}

werde ich sie doch von jetzt ab sorgfaltig notiren. Aus der Zahl derselben, verglichen mit der Grö:se des Gesichtsfeldes und der Zeit, während welcher überhaupt beobachtet wurde, lärst sich vielleicht mit der Zeit cin allgemeiner Schlufs auf die Menge dieser Körper gewinnen.

Dorpat 1842. Mai 23.

Für heute nur gelegentiiche Bemerkungen üler einige Doppelsterne, welche mir die Aufmerksamkeit der Beobachter zunächst zu verdienen scheinen. 
Drei derselben, die ich im vorigen Jahre noch länglicht erblickte, sind jetzt für das hiesige Fernrohr schon völlig rund Es sind dies

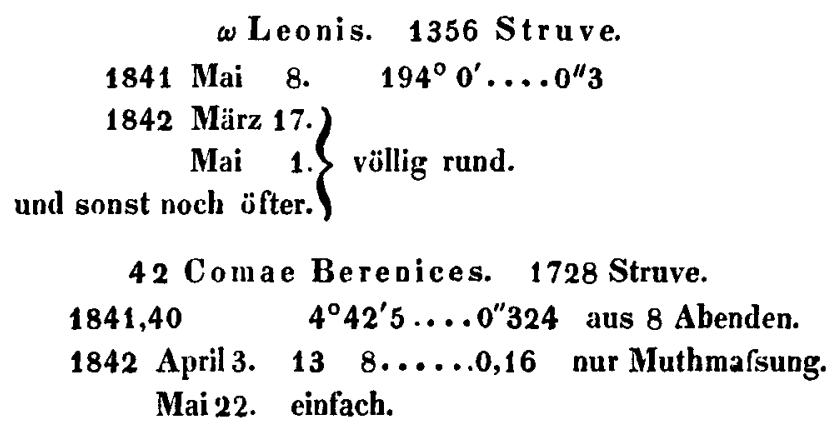

$\gamma$ Coronae. 1967 Struve. $1841,50332^{\circ} 18^{\prime} 2 \ldots 0^{\prime \prime} 184$ aus 11 Abenden. Keilförmige 1842 Mai 20 einfach.

Figur.

Der umgekehrte Fall ist eingetreten bei

$$
1663 \text { Struve. }
$$

1841 Mai 25 einfach.

$1842,33123^{\circ} 18^{\prime} 2 \ldots 0^{\prime \prime} 55$ aus 2 Ahenden. 1938 Struve (nahe bei $\mu$ Bootis)

wird vielleicht bald ein ähnliches Phänomen darbieten: er rïckt immer näher zusammen.

$$
\begin{array}{lllll}
1841,47 & 308^{\circ} 43^{\prime} 5 \ldots .0^{\prime \prime} 825 & \text { (2 Abende.) } \\
1842,40 & 305 & 41,8 & 0,70 & \text { (2 Abende.) }
\end{array}
$$

Vor 21 Jahren war die Distanz die doppelte der gegenwärtigeı.

\section{Sternbedeckungen beobachtet auf der Hamburger Sternwarte.}

1842 . R. Weyer. Funk.

Mai 12. 118 Tauri | Eintr. 9h $6^{\prime} 44^{\prime \prime} 16\left|44^{\prime \prime} 6\right| 44^{\prime \prime} 4 \mid$ M.Z.

Der Comes etwa eine Secunde früher.

Am 14ten Mai war beim Eintritte von p Geminorum die Witterung so ungünstig, dafs es sich nicht mit Bestimmtheit angeben läfst, ob der Stern hinter dem Monde oder Wolken verschwand. Herr Weyer hat den Eintritt zu $8^{\text {h }} 19^{\prime} 1^{\prime \prime} 7$ angegeben.
R. Weyer. Funk. Austr. von p Geminor. | $9^{\text {h }} 15^{\prime} 24^{\prime \prime} 05\left|9^{\text {h }} 15^{\prime} 22^{\prime \prime} 0\right| 9^{\text {h }} 15^{\prime} 25^{\prime \prime} 0$. Es ist wohl überflüssig zu bemerken, dafs vom Austritte eines Sterns $6^{r}$ Gröfse aus dem hellen Mondrande nicht dicselbe Präcision als von seinem Austritte aus dem dunkeln Rande zu erwarten steht.

Schreiben des Herrn Professors v. Boguslawski, Directors der Breslauer Sternwarte, an den Herausgeber. Breslau 1842. Mai 13.

Gestern Abend habe ich den Eintritt von 118 Tauri am dunkeln Mondrande am 43. füfs. Fraunh. mit 72mal. Vergröfserung wie ich glaube sehr gut beobachtet, wenn die Zeit auch noch einer kleinen Correction bedarf, deren (gewifs immer nur kleinen)
Betrag ich später mitzutheilen nicht verfehlen werde. Er erfolgte nach meiner Beobachtung um $12^{\mathrm{h}} 56^{\mathrm{m}} 11^{\mathrm{s}} 17 \mathrm{St} . \mathrm{Zt}$. Herr Dr. Griishang erhielt (seine erste Beobachtung dieser Art) am 4f. Fr. mit $42 \mathrm{mal}$. Vergr. $12^{\mathrm{h}} 56^{\mathrm{m}} 12^{\mathrm{s}} 44$.

v. Boguslawski.

\section{I $\mathbf{n} \mathbf{h}$ a $\mathbf{l}$ t.}

(zu Nr. 451.) Bericht über die Bihliothek der Hauptsternwarte in Pulkova, nach deren Bereicherung durch den Ankauf der Büchersammlung des verstorbenen Dr. Olbers in Bremen, nebst einigen angehängten bibliographischen Notizen. Von Sr. Excellenz dem Herin Staatsiath und Ritter $\nu$. Struve. (Beschlufs.) p. 321.

Vermischtes. p. 335.

(zu Nr. 452.) Mordsculminationen in Altona 1842. Von Herrn Th. Clausen. p. 337.

Vergleichung der Gaussischen Theorie des Erdmagnetismus, mit $A$. Ermans magnet. Beobachtungen. Von Herm H. Petersen. (Fortsetzung.) p. 341 .

Ueber die terrestrische Refraction. Von IIerrn H. H. Denzler. p. 347.

Schreiben des Herrn Hofraths Mädler, Directors der Sternwarte in Dorpat, an den Herausgeber. p. 349.

Sternbedeckungen beobachtet auf der Hamburger Sternwarte. p. 351 .
Schreiben des Herrn Professors $\nu$. Boguslawski, Directors der Breslauer Sternwarte, an den Herausgeber. p. 451. 